\title{
UNIVERSITIES AS SMART CITY DRIVERS IN SMALL AND MEDIUM-SIZED CITIES
}

\author{
L. Bodum, D. Moreno \\ Dept. of Planning, Aalborg University, Aalborg, Denmark - lbo@plan.aau.dk
}

Commission IV, WG IV/10

\begin{abstract}
KEY WORDS: Small and Medium-sized Cities, Quadruple Helix, Sustainable Development Goals, Low-cost Sensor Probes, Air Quality, Big Data
\end{abstract}

\begin{abstract}
:
Many small and medium-sized cities meet their limitations when it comes to deliver longer running smart city services and to manage the complexity of big data. There is a tendency to be stuck in pilots and smaller set-ups because the knowledge and innovation that is required for these projects and services can't be obtained in organisations, where there is more focus on daily operations and maintaining service levels in welfare. At the same time there is a need for a scientific baseline in relation to move our urban areas towards a more sustainable future. Someone needs to deliver data that can document the challenges that cities stand up against reaching the UN Sustainable Development Goals (SDG). This paper describes a case where the local university together with a research-based start-up company have taken an initiative to deploy and manage a network of multi-functional probes (MONTEM CityProbes) that contains a large number of sensors that can track a broad suite of environmental data in near real-time. The service is established with open data capabilities and with a hope to kickstart further innovations in smart city developments and to strengthen the IoT eco-system of the region. The specific example focusses on monitoring the air quality from 25 probes deployed in places with strategic importance and where there is heavy traffic intensity from fossil-fuelled motor vehicles.
\end{abstract}

\section{INTRODUCTION}

\subsection{Smart Cities in generations}

The development and maturation of the smart city concepts and framework has been happening since the term was launched more than 10-15 years ago (Kitchin, 2014). Recently it has been presented as a technology that already have been through two generations of development and is going into the third (Cohen, 2015). The first generation was driven by the larger technology companies such as IBM, Cisco and Siemens (Townsend, 2013).

The second generation was mainly driven by some innovative cities such as Barcelona, Vancouver and Singapore and the most important characteristics from this generation was the ability to change the organizations with the right amount of creativity and innovation. They are mostly larger cities and metropoles (Mora et al., 2017).

The third generation have now come into bloom and the characteristic features of this new type of smart cities are very much related to co-creation and collaborative organizations. They are driven by citizens and innovation networks where the local IoT ecosystem will play a prominent role (Cardullo and Kitchin, 2018).

\subsection{Collaboration and quadruple helix in small and medium-sized cities}

The definitions related to classification of cities was redefined by OECD and EU in 2012 (European Commision, 2012; Rosina et al., 2012). In this definition an urban centre with more than 50,000 citizens can be considered a city. The smallest cities then have between 50,000 and 100,000 citizens. Medium-sized cities have between 100,000 and 200,000 citizens. The cities that are in focus in this study therefore have less than 200,000 citizens in the urban centre.
Universities within these cities play an important role in the local innovation environment. They host incubators and create spin-off from research who in return require assistance from the research environment there (Fitjar and Gjelsvik, 2018). This dependency creates a common platform for regional innovation and networks where the local municipality also participates.

Innovation is a collaborative activity that normally involves a lot of different actors. Lately the normal triple helix model with academia, businesses and government has been extended to also include the media- and culture-based public. That is also referred to as the quadruple helix model (Carayannis and Campbell, 2009).

Third generation smart cities and the development of a quadruple helix in local innovation shapes the platform for new ways of looking at how things are done in small and mediumsized cities. The citizens can be more involved through transparency in government and access to open data portals. The municipalities can reach out to private businesses and start collaborating about the re-use of both public and private data. The universities can extend and complement their applied research and take on the responsibility for creating new IoT infrastructures which also could serve as a learning platform for their students.

\subsection{The new role of regional universities}

Following the intensified tough competition between universities for external funding on a global scale, some of the regional universities have been forced to change their strategy towards something that might relate to the similar development within the business world. A distinct profile through a smart specialization profile could be the way forward for many regional universities. 
Whether it would be with an intensified effort on education or through more applied areas within science and technology, will have to depend on the specific profile of each university.

\section{NEED FOR A SCIENTIFIC BASELINE}

\subsection{Towards the UN Sustainable Development Goals}

A general and global political consensus has been built around the UN Sustainable Development Goals (SDG) that was launched just a few years ago. The goals were presented and agreed on at a historic UN Sustainable Development Summit in New York, September 2015 (United Nations, 2015).

There are 17 main goals in the agenda towards 2030, reaching from ending poverty to revitalization of Global Partnership for Sustainable Development. Goal 17 about Global Partnership is a specific acknowledgement of the fact that the goals cannot be reached within the timeframe unless governments, civil society, the private sector and other actors such as knowledge institutions come together and mobilize the available resources.

Under each of the 17 goals there is a substantial list of targets. The total list consists of 169 targets. The purpose of the targets is to address the individual domains within the overall structure of the main goals. To operationalize the work and establish a scientific baseline for the SDG, it was decided to go even further and define a number of indicators under each target. This framework was established in the summer of 2017 and consist of 232 individual indicators (United Nations, 2017).

Each indicator has a clear data definition and the work behind the documentation of these have been headed by the UN Statistical Commission and was approved at their $48^{\text {th }}$ session in March 2017. Among other matters of this session was a continued support for the work done by an expert group to tie geospatial and statistical data closer together. In some cases, it is not possible to provide documentation for the indicators without a comprehensive use of geospatial data. The combination of geospatial and statistical data will eventually provide a stronger foundation for each of the indicators and make it possible to design a better review and follow-up of the implementation of the SDG (Scott and Rajabifard, 2017).

The following case for providing a scientific baseline for the indicators will focus on air quality and the impact it has on the well-being and environmental sustainability in cities. This could eventually touch upon many of the SDG's, but this study will particularly focus on goal 11 and goal 3 .
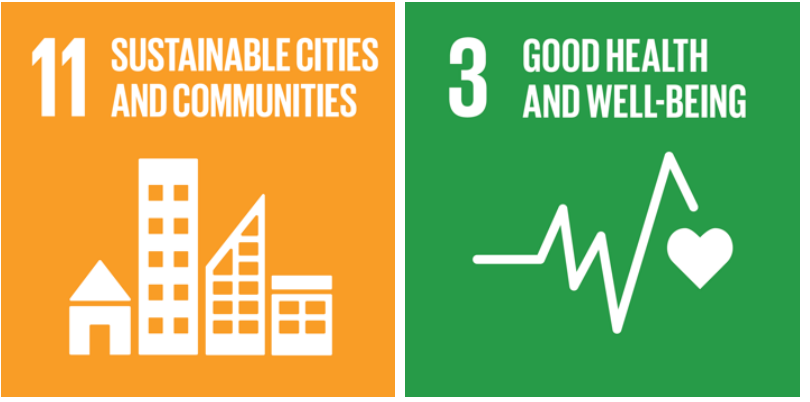

Figure 1. SDG 11 and 3, which will be in focus for the specific case in this study.
2.1.1 Goal 11: Make cities and human settlements inclusive, safe, resilient and sustainable.

This goal is one of the more comprehensive and covers a wide array of subjects. It covers housing, mobility, planning, cultural and natural heritage, resilience to disasters, environmental impact and access to green and public spaces. In relation to this study it is relevant to focus on target 11.6:

Target 11.6: By 2030, reduce the adverse per capita environmental impact of cities, including by paying special attention to air quality and municipal and other waste management

There are two main environmental elements that make up this target. The first is the management of waste from the city and the second is the air quality. The focus of this study is the air quality. The indicator that materialize this target is indicator 11.6.2:

Indicator 11.6.2: Annual mean levels of fine particulate matter (e.g. PM2.5 and PM10) in cities (population weighted)

This indicator is either calculated from a model or from ground measurements. The annual mean levels are very different for each country, but the main trend is that the level have not changed considerably since 2005 and it does not seem to fall considerably before 2030 . For the country of Denmark, the value was a little more than $12 \mu \mathrm{g}$ per $\mathrm{m}^{3}$ in 2005 and this was reduced to $10 \mu \mathrm{g}$ per $\mathrm{m}^{3}$ in 2017 . The average for the whole EU was just below $16 \mu \mathrm{g}$ per $\mathrm{m}^{3}$ in 2005 and was reduced to $13 \mu \mathrm{g}$ per $\mathrm{m}^{3}$ in 2017 (Global Burden of Disease Collaborative Network, 2017).

2.1.2 Goal 3: Ensure healthy lives and promote well-being for all at all ages.

This goal covers a long list of health-related subjects and it would be difficult to describe them all in the context of this study. Way down the list there is a target that relates to air pollution.

Target 3.9: By 2030, substantially reduce the number of deaths and illnesses from hazardous chemicals and air, water and soil pollution and contamination.

From this target comes three indicators. The first of them is a death rate related to air pollution. The second indicator is a death rate related to unsafe water, sanitation and hygiene. The third indicator is related to unintentional poisonings. This study will only focus on the first of these indicators.

Indicator 3.9.1: Mortality rate attributed to household and ambient air pollution

The indicator is in fact a combination of two elements. The first element is household air pollution (indoor) and the second is ambient air pollution (outdoor). In Denmark there was approximately 20 death per 100.000 inhabitants related to air pollution in 2005 . This number has decreased to 13.6 death per 100,000 inhabitants in 2017. On a country wide scale, it turns out there is a fall in the number of deaths related to air pollution. In EU in total there was 19.2 death per 100,000 inhabitants in 2016 related to air pollution. These numbers consist of a long list of contributing elements and one of them is the measurement of particulate matter (PM2.5) (Rictchie and Roser, 2019). 
This emphasize that the continued measurements of particulate matter (PM2.5) is an important indicator for a meaningful scientific baseline to evaluate both SDG goal 11 and 3.

\section{LOW-COST SENSOR PROBES - A CASE STUDY}

\subsection{Current situation for air quality monitoring}

The following case presents the current situation for air quality monitoring in the city of Aalborg in Denmark. At the moment the municipality of Aalborg do not have any regular activities in the field of measuring the air quality in the city of Aalborg. The only regular measurements come from the Danish Centre for Environment and Energy (DCE) at Aarhus University. They are responsible for the national monitoring program where particles and gasses are measured in the streets of the four major cities in Denmark. They also have established background stations in the same cities and furthermore in remote locations far away from any direct sources of air pollution. Finally, they also developed a spatial model of the yearly exposure generated from different data sources on each official address point in Denmark. This model shows the average value of exposure (Jensen et al., 2017)

The current situation in Aalborg is unfortunately even more limited in the monitoring since there are no measuring station on street level due to the fact that the only station was closed in 2014 when a major building construction came in the way. The plan was to establish a new location for the station not far away from the original place. But this has still not happened in medio 2019. There is a good chance that this street station will be reestablished in the near future. Nevertheless, the national program do not focus on variations and distributions of air pollution in each individual street and therefore cannot show how the local geometric conditions can amplify and condense the effects of the pollution and create urban canyons (Farrell et al., 2015).

It will be necessary to include several different methods when it comes to increase the monitoring of air quality in any city. For the specific situation in the case of Aalborg, it could be a combination of very reliable air quality measuring stations and low-cost equipment that would be either geostationary or mobile. The official monitoring stations include high profile equipment and deliver absolute measurements with calibrated results for different sizes of particulate matter (PM10 and PM2.5) and a substantial list of gasses such as $\mathrm{O}_{3}, \mathrm{NO}_{2}, \mathrm{SO}_{2}$, $\mathrm{CO}$ and $\mathrm{CO}_{2}$. For the low-cost equipment the measurements normally consist of one sensor that delivers the number of particles in either size PM10 or PM2.5 and some gas sensors that most likely will only give a relative value that needs to be calibrated up against the more expensive stations or a model that includes meteorological data.

A number of studies around the world have in recent years been focussing on the validity and quality of low-cost sensors in the air quality monitoring and exposure estimations. The conclusions from these studies all line up that there are still a lot of challenges when it comes to the performance of the low-cost sensors, but they also acknowledge the value they give to the overall modelling of real-time monitoring of air quality (Castell et al., 2018, 2017; Liu et al., 2017; McKercher et al., 2017; Schneider et al., 2017). The low-cost sensors will be geographically spread around the city and therefore covers new street sections and a larger area. This will show the variations in time and space and will give new knowledge about the distribution of air pollution in the street and above street level.

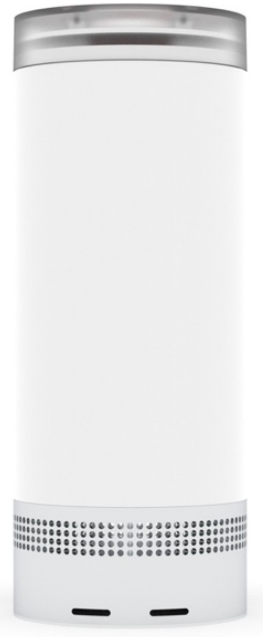

Figure 2. The MONTEM CityProbe. The height of the probe is $192 \mathrm{~mm}$ and the diameter $75 \mathrm{~mm}$. It is made from hard plastic and light metals and weighs $558 \mathrm{~g}$. The power supply comes from a solar panel attached by a cable.

The hypothesis tested in this study is that less expensive sensors deployed in the inner city can identify problematic street areas and public spaces with high concentrations of particulate matter or high levels of ozon, nitrogen oxides or carbon oxides. This is not only a matter of where the problematic areas are but also a matter of when (in the time of a week or a day) it is problematic to be present in those polluted areas.

\subsection{CityProbe from MONTEM}

The sensors, that represent the low-cost segment of air quality measuring equipment in this study are developed by a small start-up company by the name MONTEM. This company is a spin-off from Aarhus University in Denmark started by two former students recently graduated. Their product was officially launched in 2018 and is called CityProbe (MONTEM, 2019).

The CityProbe contains two sensor modules. In the bottom of the probe there is a module which contains sensors for particulate matter ( $\mathrm{PM} 2.5)$, gasses $\left(\mathrm{NO}_{2}\right.$ and $\left.\mathrm{CO}\right)$, temperature, pressure, humidity and noise level. At the top of the CityProbe there is a module which contains sensors for light intensity, UV index, rain and visual output. The visual output is several RGBLED that can illuminate the top of the probe. In all there is 11 sensors within the MONTEM CityProbe. A few of these sensors measure in an absolute value, but most of the environmental sensors actually works with a relative value, which means that it becomes important to calibrate up against official and more reliable instruments. The communication module of the CityProbe is in the study based on LTE and works with traditional $4 \mathrm{G}$ mobile networks. In future versions of the CityProbe it will be possible to upgrade the communication module to more standardised IoT communication technologies such as LoRaWAN or NarrowBand (NB).

The frequency of measurements sent from the CityProbes can be adjusted to fit the specific purpose. However, it is quite power demanding to send data with a frequency higher than 10 minutes while the CityProbe is powered on solar panels. Combined with a relatively cold climate in Denmark, the early tests showed that it could mean that the batteries were completely emptied and the CityProbes went into a power saving state. The conclusion of this dilemma was that the 
frequency of measurements would be adjusted in respect to the time of year. The way to adjust the settings of the CityProbe is through an upload of the specific version of firmware.

\subsection{Planning the deployment of CityProbes}

Aalborg University have access to 50 CityProbes from MONTEM. Half of those were deployed in the city of Aalborg and the rest works as a reserve pool from where you can quickly make substitutions or lend out a few to more specific short-term studies or projects.

When planning the deployment of the CityProbes, it was important to identify the main objectives for the study. There were actually several different interests at stake in this study. The main interest although was to identify what influence the motorized traffic had on the air quality in the streets of Aalborg. The objective of the study was in the first place to point out the most problematic crossings, stretches and intersections in the street network in regard to air quality. It was therefore decided to place the 25 CityProbes in light poles where many trucks, cars, bikes and pedestrians pass by every day.

It was also decided to cover longer stretches of roads with high traffic counts. That meant putting up CityProbes on the same street with a relatively shorter distance between them. That could give the possibility to test if the local conditions and the specific streetscape design and surrounding buildings would affect the measurements of the air quality and other environmental measurements.

After an iterative process where planning of the network was complemented with field observations of the specific locations of each station, the provisional plan for the network was finalised. This plan went into an internal hearing process within the project group and was revised with minor updates. Then an application for the local authorities was produced. This application was necessary because the light poles are placed in public areas where it is the traffic department in the municipality that is responsible for technical equipment and other material (e.g. election posters) that are mounted on and fastened to these.

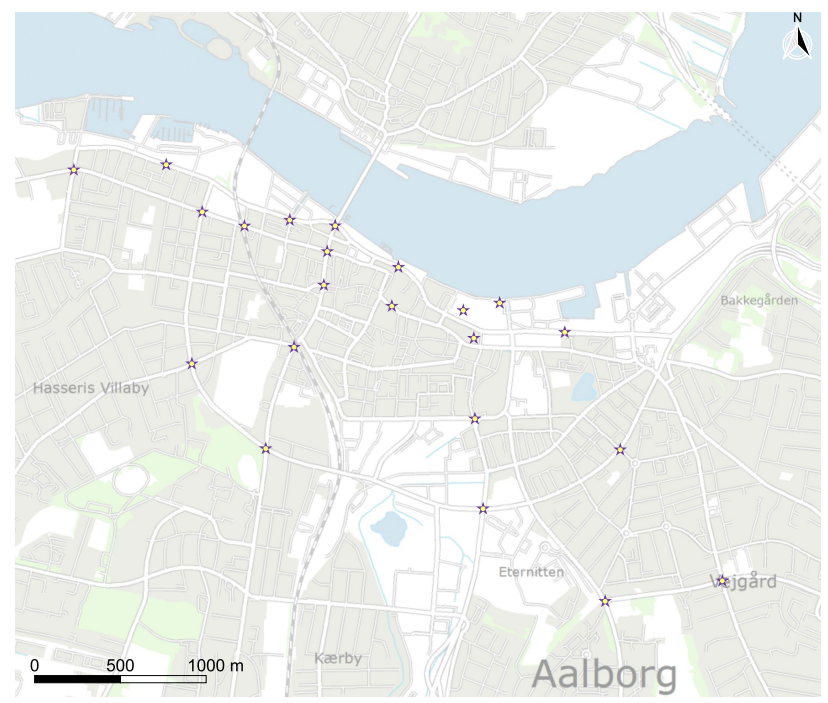

Figure 3. Map showing the location of 22 of the MONTEM CityProbes in Aalborg. The last 3 CityProbes in the network are placed in the outskirts of Aalborg where the main university campus is situated.

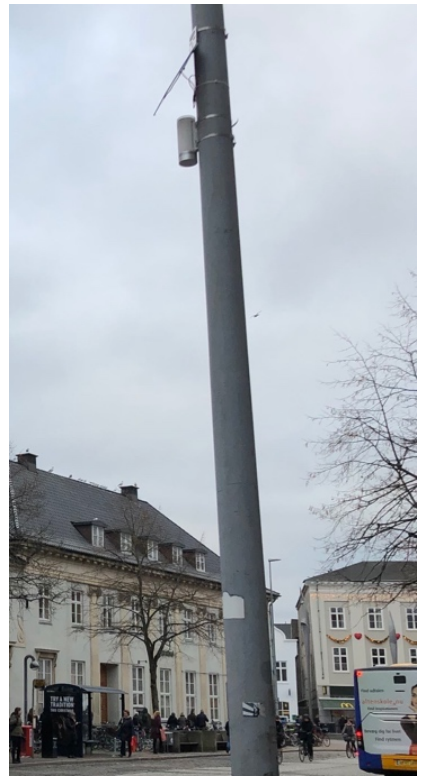

Figure 4. Station 16 in the sensor network mounted with a MONTEM CityProbe. The height of the CityProbe should be at least 4 meters.

The application was a combination of a presentation of the MONTEM CityProbes and a comprehensive list with exact positions, descriptions of the stations and a picture showing where (which light pole and how high?) the CityProbe would be mounted together with the solar panel. After considering the case for a period of two weeks the permission was given by the municipality.

Next phase of the deployment was to go around to each station and mount the CityProbes. The stations were established in the field and activated remotely through a procedure where the latest firmware is installed and the CityProbe starts communication with a status of all sensors. The small size and simple mounting procedure for the CityProbe makes it easy to change units if they are out of service or damaged. It is also fairly unproblematic to change position of each station or establish new stations if it becomes necessary to condense or reconfigure the network. Though it should be noted that replacements and establishing new stations require new permissions.

\subsection{Calibration of sensors}

Due to the way that low-cost sensors for air quality works, it is essential to have some kind of calibration up against more reliable equipment with a higher degree of reliability. This goes for the particulate matter measurements that delivers an absolute value for PM2.5 but even more importantly for the measurements of the gasses which are relative with a scale of values from 0 to 4096 .

For this reason, a bilateral agreement has been made with Danish Centre for Environment and Energy (DCE). Station 66 in the CityProbe network was established on the wall of the official background station 8158 in Aalborg. With data from this station and with meteorological information about the temperature, air pressure, wind direction and wind speed for the city, it has become possible to test the absolute values of PM2.5 and to re-calculate the relative values for $\mathrm{NO}_{2}$ to something that makes a better representation of these values for the same type of gas. 


\subsection{Dashboard for CityProbes}

A public dashboard has been developed with the purpose of maintenance of equipment and distribution of data from the CityProbes. This is a very early implementation of a dashboard and until now mainly created for internal purposes.

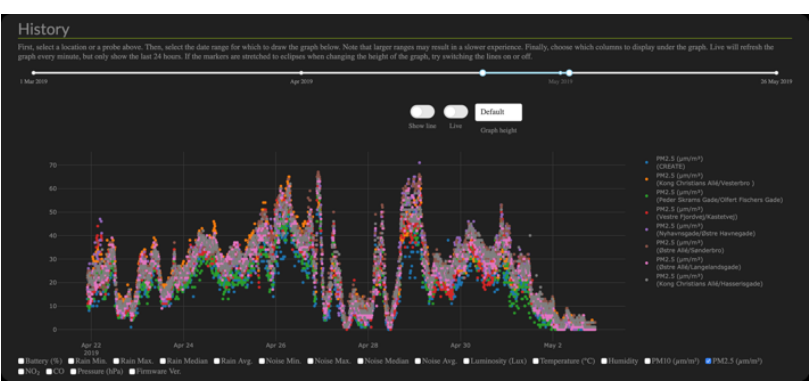

Figure 5. Example scatter diagram from the CityProbe dashboard shows PM2.5 data from 8 stations for 1 week.

The main reason for the dashboard is to provide basic service information about the CityProbes for people involved in the project. The information could be about battery levels on the CityProbes or about the status of each of the sensors in the CityProbes. You can also identify some specific data series and afterwards extract smaller datasets for further analysis. In figure 5 you can see a scatter diagram that shows values of PM2.5 for 8 stations for approximately a week. Data are in the range 0-70 $\mu \mathrm{g}$ per $\mathrm{m}^{3}$.

There will eventually be other dashboards and services developed to present the data from this network of sensors and perhaps presented together with other data delivered from other sensor networks and projects. They will all have different objectives and target groups. This is one of the outcomes from a strategy where certain data, that origins from research projects will be made openly available for business, local government and citizens. You can find the dashboard at: $\mathrm{http}: / /$ cityprobe.ciss.dk

\section{DATA MANAGEMENT}

\subsection{IoT infrastructure}

The different options for communication network platforms behind IoT impede the interoperability and makes it difficult to implement solutions that are covering several domains and business areas. It tends to keep the silos alive and makes it difficult for small and medium-sized cities to invest in the right infrastructure.

The CityProbes in this project are equipped with LTE $(4 \mathrm{G})$ as the communication protocol for wireless data transfer. This is also known as cellular IoT. When the amount of data rises and the need for better solutions for data storage evolves, it will be necessary to reassess the whole strategy and change technology towards solutions that can support big data and faster communication. There is a hope that the rollout of a $5 \mathrm{G}$ communication infrastructure will give more possibilities and solve some of these dilemmas.

The data from the CityProbes is continuously saved in a Postgres database and then available for display on the dashboard presented in section 3.5. This is also a temporary solution that will change within a considerable future.

\subsection{University Cloud Service - CLAAUDIA}

Aalborg University plans to deliver a cloud service for both research and teaching purposes. This development is wanted from many sides. First of all, it will help solve a lot of data management issues that have come up in recent years as a reaction from several research project managers. There is a need for better and more secure data storage in many research projects and it will be easier to run this service from a dedicated server. It has also become more evident with the implementation of the GDPR directive in EU that the documentation and use of personal data requires more attention also in situations where data is used in research projects.

There is also a need to support the interoperability between different research groups and make their data available across campus for students as well as for staff. If the data from the CityProbes could be used in other departments and programs it would probably create more knowledge about how the local air quality affects society, local conditions or even the personal health of the citizens in the locality where data is gathered.

The next level of interoperability would be to open access to data and solutions for everyone. That would mean a change in the way the surrounding society conceives their local university and put the universities in the driving seat for promoting smart technologies in small and medium-sized cities. It would also boost the concept of open data and open access to research and strengthen the quadruple helix concept presented in the introduction of this article. The CLAAUDIA service is under development and will be ready for implementation in early 2020.

\section{APPLICATIONS}

\subsection{Monitoring the air quality in Aalborg}

The services from the sensor network presented in this paper could be used by the city of Aalborg to monitor the air quality and make sure that the specific SDG's mentioned in chapter 2 are in focus and the more specific pollution in the form of particulate matter PM2.5 is reduced. Data for a period of 14 days $(25 / 06 / 2019$ - 09/07/2019) were downloaded from 10 stations in the network and analysed together with weather data and traffic data from the inner city of Aalborg.

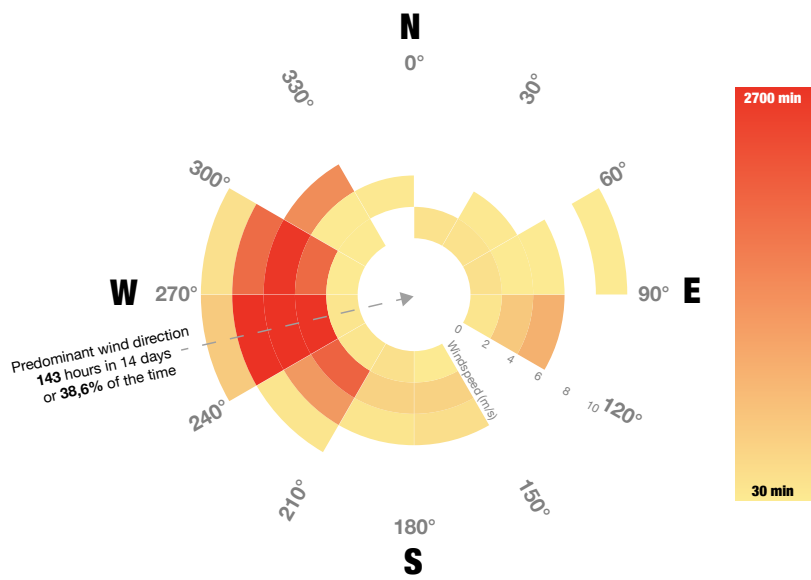

Figure 6. Wind direction and wind speed have a huge impact on the distribution of particle matter (PM). This wind rose (also known as a coxcomb chart) shows the predominant wind direction and the time of different wind speed in the period from $25 / 06 / 2019$ to $09 / 07 / 2019$. 


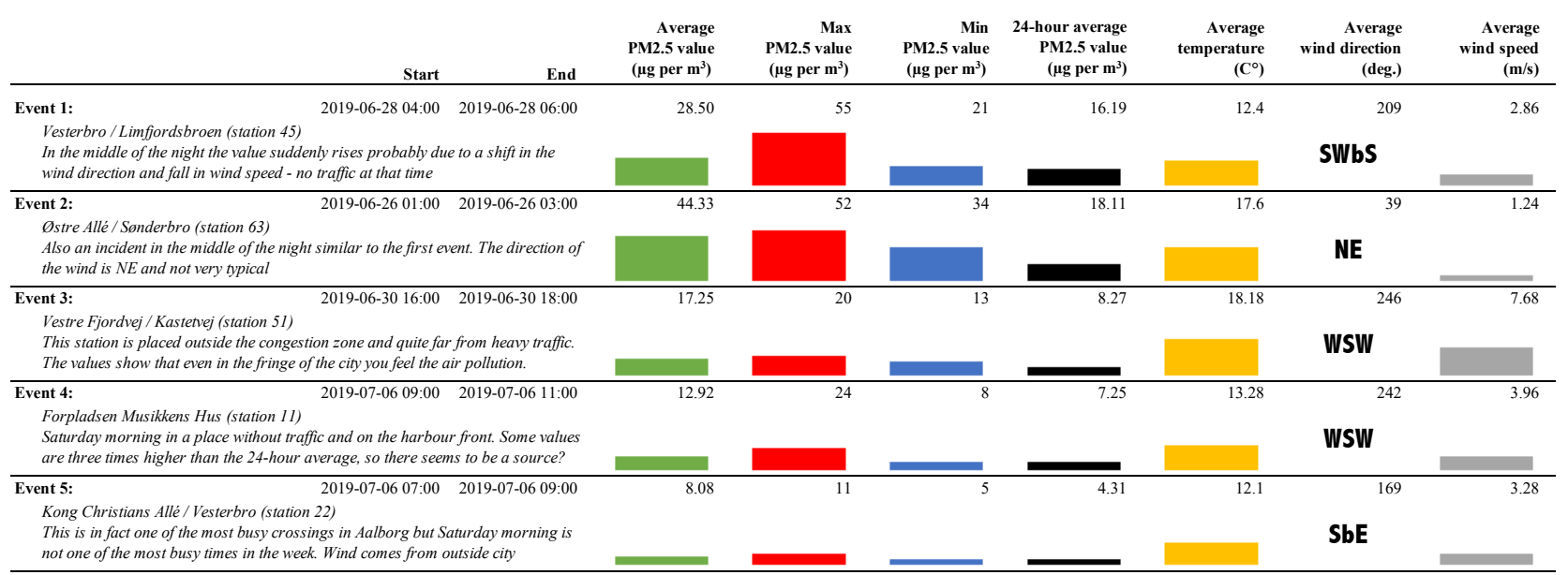

Table 1. Five events in a specific two-week period (25/6/2019 - 09/07/2019) where the level of PM2.5 is different from the normal 24-hour average value. With this report tool it will be possible to compare how different stations perform in the city and to do placebased analysis.

This could also be met through regulation and legislation if the municipality adopted tougher measures such as environmental zones where fossil fuelled motor vehicles were prohibited or severely limited. They could also introduce other measures and immediately calculate the effect by looking at the values coming from the CityProbes. Within a couple of years, it would even be possible to regulate traffic directly from the live measurements.

These precautions are already in use in many other cities around the world, where they have more comprehensive and live monitoring of the air quality. That is also the principle implemented by EU through the European Environmental Agency and their European Air Quality Index that was introduced in late 2017 (EEA, 2017).

\subsection{Student projects and hackathons}

Another obvious application could be to provide data for students that wants to work on projects related to the urban environment and how it would be possible to address specific SDG's. It would open up for innovations and new inspiration for combining different data types and do experiments with new forms of spatial analysis and visualisations. Students could work on both technical aspects as well as organisational aspects of the measurements. This could be done in case competitions or in hackathons and through open invitations to these events it could even involve students from other institutions and universities. Finally, it would provide knowledge about the learning environment around smart city solutions.

\subsection{Place-based events}

With the deployment of 25 CityProbes, each equipped with a wide range of environmental sensors, it becomes possible to study specific events, where data is outside the typical range without being a definite outlier. First of all, it is necessary to express a few reservations in regard to the technical liability of the CityProbes. They have not been tested and calibrated in function and therefore it is not possible to know the exact quality of the data. Data for a period of 14 days (25/6/2019 $9 / 7 / 2019$ ) from 10 stations in the inner city of Aalborg were downloaded and examined thoroughly. With a specific focus on PM2.5 measurements and the weather data for the same period, each station was investigated for different data.
In this study we isolated five distinct events where the PM2.5 values seemed to be higher than the typical values found in other locations, at other times of the day or with different weather conditions. It is important to stress that the number of variables and their individual dependencies would be too much to investigate in this study. This is a limited attempt to try to explain these occurrences where only a few of the variables are mentioned. In table 1 the five events are listed together with a short description and some values to show how much they differ from normal values. We found very limited evidence for a correlation between the high PM2.5 values and the wind direction and speed. This is one of the scenarios that we need to investigate further before it can be clearly confirmed.

\subsection{Time Series}

Since the data is collected with a registration of time, it seems relevant to create time series where the spatial and temporal dynamics in air quality is promoted. The hypothesis here is that local conditions (traffic, urban structure and meteorology) has a huge impact on how the air quality is changing over time. An example of this type of analysis is shown in figure 7 (Dias and Tchepel, 2018).

\section{CONCLUSIONS}

\subsection{Conclusions}

The small and medium-sized cities can be supported by their regional university when it comes to establish parts of the technical infrastructure behind a smart city. The universities can also provide the necessary resources for innovation and development of applications. This could lead to a better foundation of the quadruple helix and therefore also the support for the involvement of citizens in urban development projects.

One of the important tasks would be to provide more knowledge and facts from big data. These data are generated by IoT and smart sensors. Some of the data that are generated can be used to establish the baseline for the SDG's. There will in the coming years be more focus on evaluation of the progress towards fulfilling the SDG's based on the indicators introduced in the framework behind these. Therefore, it will be necessary to monitor the environment more closely. 


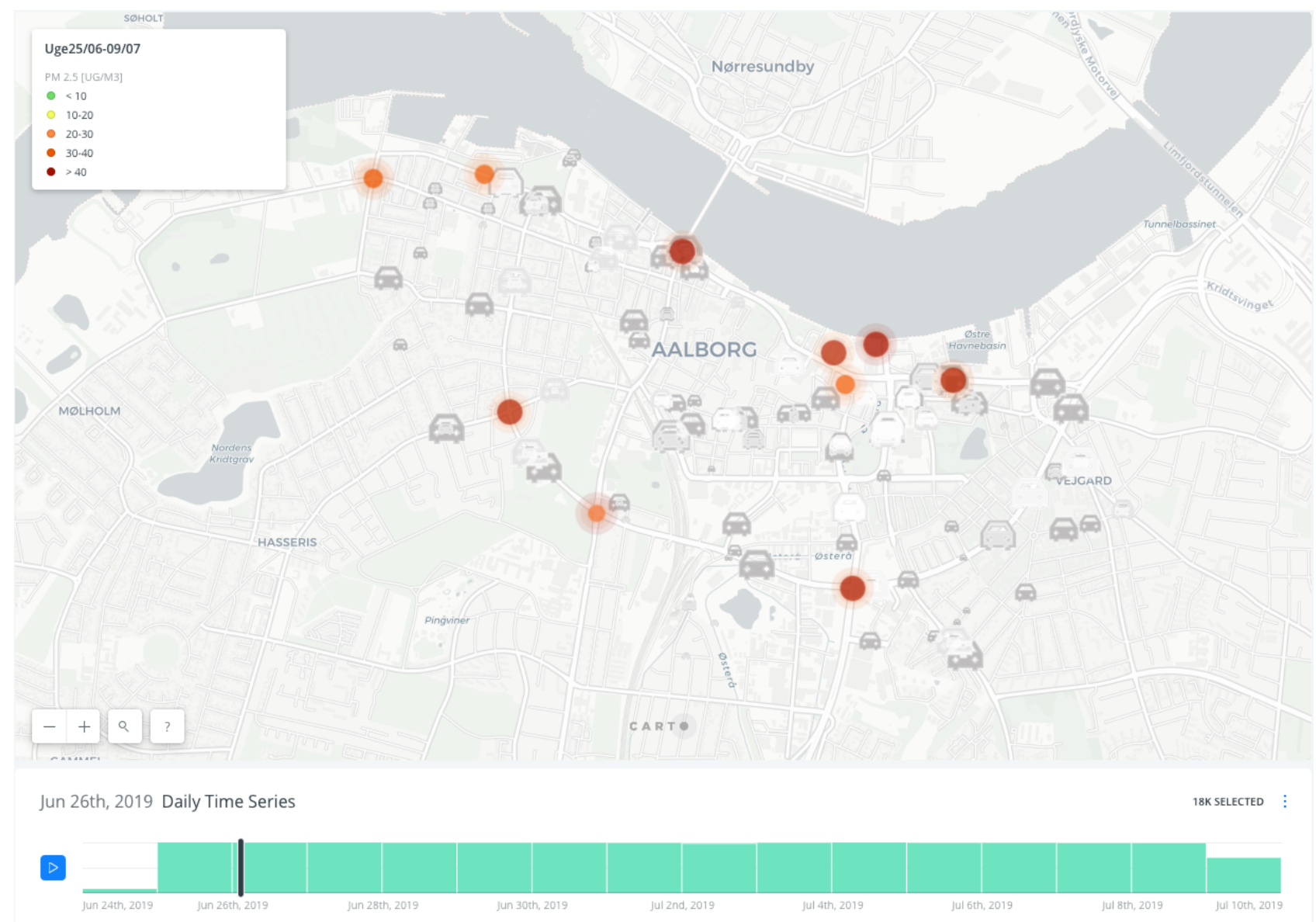

Figure 7. Time series programmed in CARTO with data from 10 CityProbes shown together with traffic data from recent traffic counts in Aalborg city. The animated version:

https://dimoreno1992.carto.com/builder/6d9c7f90-fa52-4fe3-8e0d-6a0b9fdfc2f3/embed [online] accessed 23.07.2019

A way to do this would be to establish sensors communicating through IoT and the MONTEM CityProbe has been introduced in this study as a possible technology for this purpose. 25 CityProbes have been deployed in Aalborg and data is now streaming from them and into the databases at Aalborg University. Here data will be presented through a dashboard, where it is possible to track the environmental data but also follow the technical status of the CityProbes. If there seems to be a lost connection, battery failure or individual sensors not working, it is in fact very easy to change the hardware and continue the monitoring without any long fallouts. When the analytical part of this study starts, it will be interesting to se the variations in the data material and to find the variable that is causing the variation. Is it the wind and weather or is it because of variations in traffic patterns due to time of day or week - or is it the geographical location that determines the level of particle matter or toxic gasses from the trucks, car or even cruise ships?

This study marks the start of important work where the city of Aalborg together with the university, local companies and the citizens of Aalborg will be able to check the local air quality and follow the development of this in relation to the SDG's mentioned here. It might lead to other possibilities to establish baselines for environmental indicators and initiatives to make the city both smarter and more sustainable on a quadruple helix platform.

\section{REFERENCES}

Carayannis, E.G., Campbell, D.F.J., 2009. 'Mode 3' and 'Quadruple Helix': toward a 21st century fractal innovation ecosystem. Int. J. Technol. Manag. 46(3/4), 201-234. https://doi.org/10.1504/IJTM.2009.023374

Cardullo, P., Kitchin, R., 2018. Smart Urbanism and Smart Citizenship: The Neoliberal Logic of 'Citizen-focused' Smart Cities in Europe. Environment and Planning C: Politics and Space 0, 1-22.

https://doi.org/10.1177/0263774X18806508

Castell, N., Dauge, F.R., Schneider, P., Vogt, M., Lerner, U., Fishbain, B., Broday, D., Bartonova, A., 2017. Can commercial low-cost sensor platforms contribute to air quality monitoring and exposure estimates? Environment International 99, 293302.

https://doi.org/10.1016/j.envint.2016.12.007

Castell, N., Schneider, P., Grossberndt, S., Fredriksen, M.F., Sousa-Santos, G., Vogt, M., Bartonova, A., 2018. Localized real-time information on outdoor air quality at kindergartens in Oslo, Norway using low-cost sensor nodes. Environmental Research 165, 410-419.

https://doi.org/10.1016/j.envres.2017.10.019 
Cohen, B., 2015. The 3 Generations Of Smart Cities. Fast Co. https://www.fastcompany.com/3047795/the-3-generations-ofsmart-cities [online] accessed 21.03.2019

Dias, D., Tchepel, O., 2018. Spatial and temporal dynamics in air pollution exposure assessment. International Journal of Environmental Research and Public Health 15. https://doi.org/10.3390/ijerph15030558

EEA, 2017. European Air Quality Index: current air quality information at your finger tips. Copenhagen.

https://www.eea.europa.eu/highlights/european-air-qualityindex-current [online] accessed 23.07.2019

European Commision, 2012. Cities in Europe. The new OECDEC Definition, Cities in Europe. The new OECD-EC Definition.

Farrell, W.J., Deville Cavellin, L., Weichenthal, S., Goldberg, M., Hatzopoulou, M., 2015. Capturing the urban canyon effect on particle number concentrations across a large road network using spatial analysis tools. Building and Environment 92, 328334. https://doi.org/10.1016/j.buildenv.2015.05.004

Fitjar, R.D., Gjelsvik, M., 2018. Why do firms collaborate with local universities? Reg. Stud. 52, 1525-1536.

https://doi.org/10.1080/00343404.2017.1413237

Global Burden of Disease Collaborative Network, 2017. Global Burden of Disease Study 2016 (GBD 2016) Health-related Sustainable Development Goals (SDG) Indicators 1990-2030. Institute for Health Metrics and Evaluation (IHME), Seattle, United States. https://vizhub.healthdata.org/sdg/ [online] accessed 23.07.2019

Jensen, S.S., Ketzel, M., Becker, T., Christensen, J., Brandt, J., Plejdrup, M., Winther, M., Nielsen, O.K., Hertel, O., Ellermann, T., 2017. High resolution multi-scale air quality modelling for all streets in Denmark. Transportation Research Part D: Transport and Environment 52, 322-339.

https://doi.org/10.1016/j.trd.2017.02.019

Kitchin, R., 2014. The real-time city? Big data and smart urbanism. GeoJournal 79, 1-14.

https://doi.org/10.1007/s10708-013-9516-8

Liu, D., Zhang, Q., Jiang, J., Chen, D.R., 2017. Performance calibration of low-cost and portable particular matter (PM) sensors. Journal of Aerosol Science 112, 1-10. https://doi.org/10.1016/j.jaerosci.2017.05.011

McKercher, G.R., Salmond, J.A., Vanos, J.K., 2017. Characteristics and applications of small, portable gaseous air pollution monitors. Environmental Pollution 223, 102-110. https://doi.org/10.1016/j.envpol.2016.12.045

MONTEM, 2019. CityProbe - MONTEM.

https://www.montem.io/cityprobe [online] accessed 21.05.2019

Mora, L., Bolici, R., Deakin, M., 2017. The First Two Decades of Smart-City Research: A Bibliometric Analysis. Journal of Urban Technology 24, 3-27.

https://doi.org/10.1080/10630732.2017.1285123

Ritchie, H., Roser, M., 2019. Air Pollution. Our World in Data. https://ourworldindata.org/air-pollution [online] accessed 23.07.2019
Rosina, K., Sanchez-Serra, D., Brezzi, M., Piacentini, M., Rosina, K., Sanchez-Serra, D., 2012. Redefining urban areas in OECD countries, in: Redefining "Urban": A New Way to Measure Metropolitan Areas. OECD Publishing, Paris, pp. 1958. https://doi.org/10.1787/9789264174108-4-en

Schneider, P., Castell, N., Vogt, M., Dauge, F.R., Lahoz, W.A., Bartonova, A., 2017. Mapping urban air quality in near realtime using observations from low-cost sensors and model information. Environment International 106, 234-247. https://doi.org/10.1016/j.envint.2017.05.005

Scott, G., Rajabifard, A., 2017. Sustainable development and geospatial information: a strategic framework for integrating a global policy agenda into national geospatial capabilities. Geospatial Information Science 20(2), 59-76.

https://doi.org/10.1080/10095020.2017.1325594

Townsend, A.M., 2013. Smart cities: big data, civic hackers, and the quest for a new utopia. W. W. Norton \& Company, Inc., New York, USA.

United Nations, 2017. Work of the Statistical Commission pertaining to the 2030 Agenda for Sustainable Development (A/RES/71/313), Annex (6 July 2017).

United Nations, 2015. Transforming our World: The 2030 Agenda for Sustainable Development - A/RES/70/1. New York, USA. 